Published in Tissue and Cell 36: 245-248, 2004

\title{
About the T-system in the myofibril-free sarcoplasm of the frog muscle fibre \\ Tilman Voigt ${ }^{\mathrm{a}, *}$, Wolfgang Dauber ${ }^{\mathrm{b}}$ \\ a Institute of Anatomy, University of Fribourg, Rte Albert-Gockel 1, CH-1700 Fribourg, Switzerland ${ }^{\mathrm{b}}$ Institute of Anatomy, Eberhard-Karls-University Tuebingen, D-72074 Tuebingen, Germany \\ Received 6 May 2003; received in revised form 31 January 2004; accepted 5 March 2004
}

\section{Abstract}

Previous investigations of the T-system in skeletal muscle fibres described the inter-myofibrillar relationships between T-tubules and the sarcoplasmic reticulum. They disregarded the arrangement of the T-system in the myofibril-free sarcoplasm in the area of muscle fibre nuclei. In the present investigation, the T-system was filled by means of lanthanum incubation and the myofibril-free sarcoplasm was ultrastructural examined by means of thin $(\leq 100 \mathrm{~nm})$ as well as thick sections $(>300 \mathrm{~nm}-1 \mu \mathrm{m})$ with the electron microscope. The investigation of thick sections revealed that $\mathrm{T}$-tubules meander through this myofibril-free sarcoplasm and tangle up at the poles of muscle fibre nuclei and in the area of fundamental nuclei of the motor end plate.

They are, far from myofibrils, in proximity to these nuclei, the Golgi apparatus and mitochondria. On basis of this proximity and their openings at the muscle fibre surface, a contribution at the drainage of metabolic products and at the local calcium control is discussed.

Keywords: T-tubules; Myonuclei; Motor end plate; Lanthanum

\section{Introduction}

In previous investigations the three-dimensional structure of T-system between the myofibrils of skeletal muscle fibres of mammals and frogs is described. The function of the T-system with respect to the excitation contraction coupling is in large part known (Peachey and Franzini-Armstrong, 1983). The investigation of T-system in the motor end plate (MEP) is complicated, since an ultrastructural distinction, between the sarcoplasmic reticulum and the T-system, is only possible if one of the membrane systems can be marked (Peachey, 1965). After Lanthanum incubation, the $\mathrm{T}$-system, its three-dimensional configuration and the connections of T-tubules with subsynaptic folds MEP, can be differentiated unambiguously (Dauber et al., 2000; Voigt et al., 2000). These T-tubules, which meander in the sole plate sarcoplasm, resemble more to the longitudinal oriented segments of the T-system between myofibrils than to the transversal oriented segments of the T-system between the SR-cisterns. It is probable that both segments of T-system have different functions as glycerol shock experiments

* Corresponding author. Fax: +41-263009733

E-mail address: tilman.voigt-gawatz@unifr.ch (T. Voigt).

0040-8166

doi:10.1016/j.tice.2004.03.004 with frog muscle fibres let divine. In these experiments, a preponderant vacuole formation of the T-system in the longitudinal direction of the fibre axis was observed with the light microscope (Krolenko, 1969). In addition, an accumulation of vacuoles in the perinuclear sarcoplasm of muscle fibre nuclei was described (Krolenko et al., 1995). But a distinction between $\mathrm{T}$-tubules in the perinuclear sarcoplasm of muscle fibre nuclei and sole plate nuclei was not done.

The aim of the present ultrastructural investigation is to show possible structure particularities of the T-system of frog muscle fibres in the perinuclear sarcoplasm of muscle fibre nuclei and sole plate nuclei by means of thick $(>300 \mathrm{~nm}-1 \mu \mathrm{m})$ and thin $(\leq 100 \mathrm{~nm})$ sections.

\section{Materials and methods}

The MEPs of the sartorius muscle of adult frogs (Xenopus laevis, $n=3$; Rana temporaria $n=3$ ) were examined. The frogs were anaesthetized with MS222 (Sandoz Co.) and decapitated. The legs were exarticulated at the pelvic joint. In a petri dish filled with $2.5 \%$ glutaraldehyde in $0.1 \mathrm{M}$ cacodylate buffer, the skin of the leg was removed, the superficial sartorius muscle exposed, and its fascia com- 


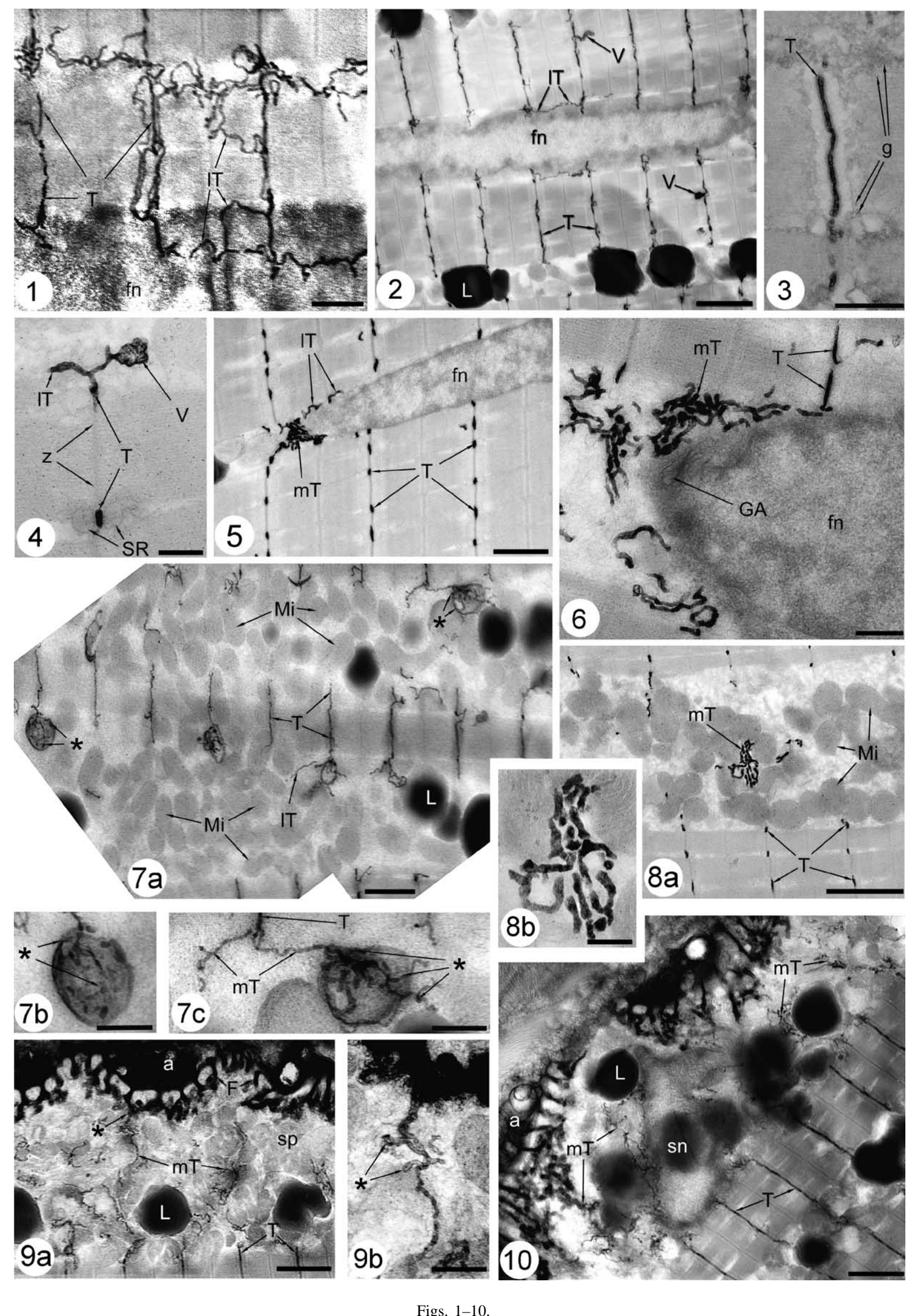


pletely removed. Then the entire leg was immersion-fixed in the same fixative for $1 \mathrm{~h}$. Subsequently, the muscles were divided into small samples and were fixed in the same fixative agent for additional $2 \mathrm{~h}$. After washing in cacodylate buffer and postfixing for three hours in $1 \%$ osmium in $0.8 \%$ potassium ferrocyanide in $0.1 \mathrm{M}$ cacodylate buffer, the tissue samples were incubated for $1.5 \mathrm{~h}$ in $1 \%$ lanthanum nitrate in $0.1 \mathrm{M}$ cacodylate buffer on an agitator at room temperature. Following dehydration by means of an ascending series of alcohol solutions, the tissue was embedded in EPON. Ultrathin sections $(\leq 100 \mathrm{~nm})$ and thicker sections (>300 nm-1 $\mu \mathrm{m}$ ) were examined in the unstained state with the electron microscope Leo912 Omega (Zeiss).

\section{Results}

The investigation of both frog species shows that the $\mathrm{T}$-system can be depicted within muscle fibres by means of the lanthanum incubation (Figs. 1 and 2). The T-system can be distinguished from the other cell organelles and myofibrils due to the contrast of the lanthanum precipitate (Figs. 1-4). In addition, the glycogen is not outlined as dark electron-dense granules (Fig. 3). With increasing section thickness, lipid droplets look like dark electron-dense masses (Figs. 2, 7, 9 and 10). In longitudinal thick sections of muscle fibres, the frequency to outline the longitudinal T-system in greater continuity is increased (Figs. 1 and 2). In such sections vacuole-like expansions of T-tubules, which extend in both directions of the longitudinal fibre axis, can be shown frequently (Figs. 2 and 4). Longitudinal
T-tubules, with remarkable loop formations and diameter fluctuations, meander along the surface of muscle fibre nuclei (Figs. 1, 2 and 5). In the MEP, these meandering $\mathrm{T}$-tubules are in proximity to subsynaptic folds (Figs. 9b and 10) and are connected with T-tubules of myofibrils (Figs. 9 and 10). The sarcoplasm between myofibrils and at the nuclear poles includes a fine-meshed network of T-tubules (Figs. 5-8), which is in proximity to the Golgi apparatus (Fig. 6). This network shows local swellings, as the longitudinal inter-myofibrillar T-system. The agglomeration of tangled T-tubules in proximity to mitochondria give the impression of a net-like enclosure of individual mitochondria (Fig. $7 b$ and c). Concerning their dimensions $(\varnothing \mathrm{ca} .50 \mathrm{~nm})$ and form variability, the tangled T-tubules agree with the longitudinal T-system between myofibrils.

\section{Discussion}

The ultrastructural investigation has demonstrated that in thick sections $(>300 \mathrm{~nm})$ of lanthanum incubated skeletal muscle fibres the T-system can be shown in great expansion. The necessary structure preservation is better at frogs than at mammals (Sonoda et al., 1993). The membranes of cell organelles are more contoured and lipid droplets are more blackened at frogs than at mammals, due to the potassium ferrocyanide osmium tetroxide postfixation, which is essential for the lanthanum incubation. The contrast of glycogen, which is well outlined after the potassium ferrocyanide osmium tetroxide postfixation (De Bruijn, 1973), is reduced by the lanthanum incubation, so that the glycogen-filled ar-

Fig. 1. Thick longitudinal section of a skeletal muscle fibre with muscle fibre nucleus (fn). Because of the lanthanum incubation the T-system (T) is depicted inside the muscle fibre. Between myofibrils meandering net-like connected T-tubules (IT) spread out. Xenopus laevis; unstained $1 \mu \mathrm{m}$ thick section; bar $=1 \mu \mathrm{m}$. Fig. 2. Semithin longitudinal section of a skeletal muscle fibre. The longitudinal T-system (IT), here in proximity to a muscle fibre nucleus (fn), is sporadically intersected. Smaller vacuoles filled with lanthanum are outlined (V). Glycogen can not be shown in the inter-myofibrillar sarcoplasm; lipid droplet (L); transverse T-System (T). Xenopus laevis; unstained $300 \mathrm{~nm}$ thick section; bar $=2 \mu \mathrm{m}$. Fig. 3. Ultrathin longitudinal section of a triad, whose $T$ - tubule (T) is filled with lanthenum. At this section thickness the longitudinal T-tubules are seldom intersected. The glycogen can be hown as , 列 (V); locins is cong Longitudinal ultrathin section of a skeletal muscle fibre with a muscle fibre nucleus (fn). T-tubules (mT) are tangled up in the sarcoplasm at the nuclear pole; transverse T-tubules (T), longitudinal T-tubules (IT). Rana temporaria; unstained $100 \mathrm{~nm}$ thick section; bar $=1 \mu \mathrm{m}$. Fig. 6. Longitudinal ultrathin section of a skeletal muscle fibre with muscle fibre nucleus (fn). A Golgi apparatus (GA) at the nuclear pole is in proximity to tangled T-tubules (mT). Glycogen granules in the region of the triads are not stained; transverse T-tubules (T). Rana temporaria; unstained $100 \mathrm{~nm}$ thick section; bar $=500 \mathrm{~nm}$. Fig. 7a Longitudinal thick section of a myofibril-free sarcoplasm area in the inside of a skeletal muscle fibre. Several tangled T-tubules $(*)$ are between mitochondria (Mi); transverse T-tubules (T), longitudinal T-tubules (IT), lipid droplets (L). Xenopus laevis; unstained $500 \mathrm{~nm}$ thick section; bar $=2 \mu \mathrm{m}$; Fig. 7b a tangled T-tubule $(*)$ runs around a mitochondria. Unstained $500 \mathrm{~nm}$ thick section; bar $=500 \mathrm{~nm}$; Fig. $7 \mathrm{c}$ a transverse T-tubule (T) continuously changes in a meandering T-tubule $(\mathrm{mT})$, that tangles up around a mitochondria $(*)$. Unstained $500 \mathrm{~nm}$ thick section; bar $=500 \mathrm{~nm}$. Fig. 8 a. Longitudinal semithin section of a myofibril-free sarcoplasm area, which extends over several sarcomers in the longitudinal fibre axis. Tangled T-tubules $(\mathrm{mT})$ are between mitochondria (Mi); transverse T-Tubule (T). Xenopus laevis; unstained $300 \mathrm{~nm}$ thick section; bar $=2 \mu \mathrm{m}$; Fig. $8 \mathrm{~b}$ unstained $300 \mathrm{~nm}$ thick section $\mathrm{bar}=250 \mathrm{~nm}$. Fig. 9a. Longitudinal thick section of a MEP. Both, the extracellular area of the subsynaptic folds (F) and the T-system of sole plate are filled with ( ( ) The 1 un which approches the subsynaptic fold, repeatedly enits. Xean not be resolved up exacly, becase of the section, bar $=250 \mathrm{~nm}$. Fig. 10. Longitudinal thick section of a MEP in the area of a fundamental nuclei (sn). Next to the transverse T-system (T) the extra cellular space of the subsynaptic folds is filled with lanthanum; axon (a). Meandering net-like linked T-tubules (mT) spread out in the sole plate area; lipid droplet (L). Xenopus laevis; unstained $1 \mu \mathrm{m}$ thick section; bar $=2 \mu \mathrm{m}$. 
eas around triads and in the myofibril-free sarcoplasm look bright.

In the unincubated status, the longitudinal oriented T-tubules between myofibrils can be traced in greater expansion only by means of inconvenient reconstructions of serial sections (Peachey, 1965; Eisenberg, 1972). With the lanthanum incubation, these longitudinal oriented T-tubules as well as the T-tubules in the myofibril-free sarcoplasm can be effortlessly depicted. In this myofibril-free sarcoplasm of skeletal muscle fibres of the frog tangled T-tubules spread around the poles of fibre nuclei, the mitochondria as well as in proximity to sole plate nuclei. At the mammal, however, a similar tangled T-tubular network can be recognized only in the sole plate sarcoplasm (Dauber et al., 2000). In the literature, known to us, we could not find any statements about similar looking agglomerations of T-tubules in these areas. Reason for that might be on the one hand a too selective orientation of previous investigations with regard to the organization of the T-system between the myofibrils, to the other the assignment problem between SR and T-system (Peachey, 1965), and finally investigations by means of too thin sections.

Furthermore, as in other animal species too, vacuoles standing in touch with T-system are shown (Leeson, 1977; Forbes and Sperelakis, 1980). But at mammals, we could not show such vacuoles between myofibrils (Dauber et al., 2000).

At frogs, vacuolisations are described in the area of the Z-stripes after overloading exercise (Krolenko, 1969; Fraser et al., 1998; Krolenko et al., 1998; Nagesser et al., 1994; Gonzalez Serratos et al., 1978). A favoured vacuolisation in the longitudinal part of the T-system (Fraser et al., 1998) and at the poles of muscle fibre nuclei (Krolenko et al., 1998) could be attributed to a mechanical stabilization of the triad (Dulhunty, 1989). Next to the contribution of the T-system to the electrochemical coupling, also the drainage of metabolic products i.e. lactate is discussed as function of the T-system (Nagesser et al., 1994). Because of the proximity of the tangled T-tubules to the nuclei and Golgi apparatus, also a shortened transport pathway can be thought of in this context.

Since the T-system serves as source of inositol-trisphosphate (Carrasco et al., 1988; Volpe et al., 1986), which can trigger $\mathrm{Ca}^{2+}$-waves, the T-system could be shared at the regulation of the perinuclear $\mathrm{Ca}^{2+}$-release (Jaimovich et al., 2000; Powell et al., 2001) in those muscle fiber regions where the nuclei are far a way from myofibrils.

\section{References}

Carrasco, M.A., Magendzo, K., Jaimovich, E., Hidalgo, C., 1988. Calcium modulation of phosphoinositide kinases in transverse tubule vesi- cles from frog skeletal muscle. Arch. Biochem. Biophys. 262, 360-

Dauber, W., Voigt, T., Härtel, X., Meyer, J., 2000. The t-tubular network and its triads in the sole plate sarcoplasm of the motor end-plate of mammals. J. Muscle Res. Cell Motil. 21, 443449.

De Bruijn, W.C., 1973. Glycogen, its chemistry and morphologic appearance in the electron microscope. I. A modified $\mathrm{OsO} 4$ fixative which selectively contrast glycogen. J. Ultrastruct. Res. 42, 29-50.

Dulhunty, A.F., 1989. Feet, bridges, and pillars in triad junctions of mammalian skeletal muscle: their possible relationship to calcium buffers in terminal cisternae and T-tubules and the excitation-contraction coupling. J. Membr. Biol. 109, 73-83.

Eisenberg, B.R., 1972. Tree Dimensional branching of the T System in frog sartotius muscle. J. Cell Biol. 55, 68a.

Forbes, M.S., Sperelakis, N., 1980. Membrane systems in skeletal muscle of the lizard Anolis carolinensis. J. Ultrastruct. Res. 73, 245261.

Fraser, J.A., Skepper, J.N., Hockaday, A.R., Huang, C.L.H., 1998. The tubular vacuolation process in amphibian skeletal muscle. J. Muscle Res. Cell Motil. 19, 613-629.

Gonzalez Serratos, H.G. Somlyo, A. V, McClellan, G., Shuman, H., Borrero, L., Somlyo, A.P., 1978. Composition of vacuoles and sarcoplasrero, L., Somlyo, A.P.,
mic reticulum in fatigued muscle: electron probe analysis. Proc. Natl. mic reticulum in fatigued muscle:

Acad. Sci. U.S.A. 75, 1329-1333.
Jaimovich, E., Reyes, R., Liberona, J.L., Powell, J.A., 2000. IP3 receptors, Jaimovich, E., Reyes, R., Liberona, J.L., Powell, J.A., 2000. IP3 receptors,
IP3 transients, and nucleus-associated Ca2+ signals in cultured skeletal IP3 transients, and nucleus-associated Ca2+ signals in cult
muscle. Am. J. Physiol. Cell Physiol. 278, C998-C1010.

Krolenko, S.A., 1969. Changes in the T-system of muscle fibers under the influence of influx and efflux of glycerol. Nature 221, 966968.

Krolenko, S.A., Amos, W.B., Brown, S.C., Tarunina, M.V., Lucy, J.A., 1998. Accessibility of T-tubule vacuoles to extracellular dextran and DNA: mechanism and potential application of vacuolation. J. Muscle Res. Cell Motil. 19, 603-611.

Krolenko, S.A., Amos, W.B., Lucy, J.A., 1995. Reversible vacuolation of the transverse tubules of frog skeletal muscle: a confocal fluorescence microscopy study. J. Muscle Res. Cell Motil. 16, 401-411. microscopy study. J. Muscle Res. Cell Motil. 16, 401-411.
Leeson, T.S., 1977. Sarcotubules and subsarcolemmal caveolae and their continuity with the sarcolemma in frog striated muscle. Am. J. Anat. $150,185-191$

Nagesser, A.S., van der Laarse, W.J., Elzinga, G., 1994. Lactate efflux from fatigued fast-twitch muscle fibres of Xenopus laevis under various extracellular conditions. J. Physiol. 481, 139-147.

Peachey, L.D., 1965. The sarcoplasmic reticulum and transverse tubules of the frog's sartorius. J. Cell Biol. 25, 209-231.

Peachey, L.D., Franzini-Armstrong, C., 1983. Structure and function of membrane systems of skeletal muscle cells. In: Peachey, L.D., Adrian, R.H., Geiger, S.R. (Eds.), Handbook of Physiology. American Physiologic Society, Bethesda, Maryland, pp. 23-71.

Powell, J.A., Carrasco, M. Ad M., Estrada, M., Jaimovich, E., 2001. IP3 receptor function and localM., Estrada, M., Jaimovich, E., 2001. IP3 receptor function and local-
ization in myotubes: an unexplored Ca2+ signaling pathway in skeletal

muscle. J. Cell Sci. 114, 3673-3683.
Sonoda, M., Moriya, H., Shimada, Y., 1993. Fine structure of transverse tubules and the sarcoplasmic reticulum at the myotendinous junction of stretched muscle fibers of the rat. Microsc. Res. Tech. 24, 281-286.

Voigt, T., Dauber, W., Härtel, X., Schönemann, R., 2000. On the connections between the T-System and the subsynaptic folds in the motor end plate of amphibians. J. Neurocytol. 30, 157-164.

Volpe, P., Di Virgilio, F., Pozzan, T., Salviati, G. 1986 Role of Inositol 1,45-trisposphate in excitation-contraction coupling in skeletal-muscle. FEBS Lett. 197, 1-4. 